\title{
Editorial
}

\section{John Wickham Steeds, Holweck Prize 1996}

On the 31st of May 1996, in the amphitheatre of the University of Lille, the Holweck Prize 1996 was officially attributed to Professor John Steeds of the University of Bristol by Professor Marianne Lambert, President of the French Physical Society. The Holweck Prize is one of the most important prizes awarded annually by this Society. This prize is the joint responsibility of the French Society and the British Institute of Physics and is attributed to a French physicist by the Institute of Physics and vice versa on alternate years, thus confirming the long tradition of friendship between French and British physicists. In 1996 the choice was to be made by the French side and the designation of John Steeds was a particular pleasure for us as it recognized one of the Editors of the Journal Microscopy, Microanalysis, Microstructures as a prominent contributor in the field of Condensed Matter Physics.

Born in London in 1940, John Steeds obtained his Ph.D. in Cambridge, in the famous group of Prof. Peter Hirsch at the Cavendish Laboratory, under the supervision of Dr. Archie Howie. His research work at that time relied heavily on the use of transmission electron microscopy for the investigation of dislocation configurations in deformed monocrystals. It constituted the starting point for his long and permanent efforts to develop new tools for elucidating the crystalline structure of materials in the immediate neighbourhood of crystal defects.

Appointed Professor of Physics in Bristol, he joined another famous centre for condensed matter research associated with the names of N. Mott and C. Frank in particular. He initiated there his work on convergent beam diffraction techniques using focused electron beams in the electron microscope. It is unnecessary to describe for the readers of our Journal the basic principles and the successes of this technique for the determination of local crystalline structure and symmetry. Many papers published over the last few years in Microscopy, Microanalysis, Microstructures rely extensively on the pioneering work of John Steeds and his coworkers in this field.

Through all his contributions, which have promoted electron diffraction crystallography as a very powerful technique for the structural characterization of solids at the nanometre scale, he has established the Bristol School as a leading centre in electron microscopy. This recognition is due not only to the quality and originality of the studies performed in Bristol, but also to its wide spread influence, the result of numerous theoretical and practical training schools and workshops and a long list of publications (research papers as well as monographs, compilations, a diffraction pattern atlas...) referred to and abundantly used in every electron microscopy laboratory all round the world.

It is therefore a great honour for our community deeply involved in developing and testing new methods relying on microscopy, diffraction and spectroscopy techniques in materials science, to have one of our leaders in the field distinguished by such an award where his name will follow a long list of recipients in all areas of physics, among whom several have later won a Nobel Prize: L. Néel, A. Kastler, M. Ryle, P.G. de Gennes, D. Gabor, B. Josephson, A. Hewish. We are therefore very pleased to offer John Steeds our congratulations for his success and we hope that this recognition will confirm the importance of the topics that justify the existence of our Journal in the wider field of condensed matter physics. 